\title{
ESTUDIO PALEOAMBIENTAL DE NUEVOS YACIMIENTOS DE PLANTAS Y VERTEBRADOS DE LA "UNIDAD INTERMEDIA" DEL MIOCENO MEDIO DE LA CUENCA DE MADRID
}

Trabajo presentado a las XVIII Jornadas de Paleontología y

II Congreso Ibérico de Paleontología

Universidad de Salamanca

Salamanca, 24-29 de Septiembre de 2002

\author{
M. ${ }^{a}$ Teresa FERNÁNDEZ MARRÓN ${ }^{1}$, José Fernan- \\ do FONOLLÁ OCETE ${ }^{1}$, Carmén SESÉ BENITO ${ }^{2}$ y \\ Juan Carlos JIMÉNEZ RODRIGO
}

\begin{abstract}
${ }^{1}$ Departamento de Paleontología. Facultad de Ciencias Geológicas, UCM y Departamento de Paleontología del Instituto de Geología Económica (CSICUCM). 28040 Madrid. emarron@geo.ucm.es

${ }^{2}$ Departamento de Paleobiología. Museo Nacional de Ciencias Naturales. CSIC. C/ José Gutierrez Abascal, 2, 28006 Madrid. c.sese@mncn.csic.es

${ }^{3} \mathrm{C} /$ Bancaleros $\mathrm{n}^{\circ}$ 8. 45600 Talavera de la Reina. Toledo
\end{abstract}

Fernández Marrón, M. T., Fonollá Ocete, J. F., Sesé Benito, C. y Jiménez Rodrigo, J. C. 2004. Estudio paleoambiental de nuevos yacimientos de plantas y vertebrados de la "Unidad Intermedia" del Mioceno Medio de la cuenca de Madrid. [Palaeoenvironmental study of new plants and vertebrate sites of the "Unidad Intermedia" from Middle Miocene of Madrid basin.] Revista Española de Paleontología, 19 (2), 199-213. ISSN 02136937.

\begin{abstract}
Some palaeobotanical assemblages (spores, pollen, leaves, seeds and fruits) and microvertebrates found in the "Unidad intermedia" from middle Miocene deposits of the Madrid basin are studied.

The fossil plants appear in five sites, and the vertebrate fossils in other site, near the formers, but stratigraphically above these. The palynological assemblages are quite similar in all the sites, but the macroremains associations present taphonomic peculiarities in some of them. The fossil plants identified show some elements of acuatic, flooded, riparian and of river banks environments with some deciduous and evergreen forest taxa. The palaeobotanical data are symbolized and ecologically located in a syntetic section and in a diagram. The different environments which had been delimited are represented.

The vertebrate fossil assemblage is made of two taxa of Reptilia (one Lacertidae and one Anguidae), and, among the mammals, the Sciuridae Heteroxerus cf. rubricati, the Gliridae cf. Armantomys sp., the Cricetidae Megacricetodon collongensis - crusafonti, one Insectivora and some remains of Lagomorpha. From a biostratigraphical point of view, this faunal assemblage may corresponds to the Middle Miocene, to the Upper Aragonian, but not to latest among the Zones $F, G_{1}$ and $G_{2}$ (sensu Daams and Freudenthal, 1988a) that can be correlated with the NM 6 Unit (sensu Mein, 1975). This fauna suggests a mainly open environment and a relatively dry climate.
\end{abstract}

Key woords: Palaeobotany, Palynology, macroflora, fossil vertebrates, Miocene, Madrid basin.

\section{RESUMEN}

Se dan a conocer los vegetales fósiles (macrorrestos foliares, frutos y palinomorfos) y una asociación de microvertebrados encontrados en yacimientos descubiertos en la "Unidad Intermedia" del Mioceno de Madrid.

Los restos paleobotánicos y palinológicos se encuentran en cinco yacimientos, mientras que los de microvertebrados aparecen en otro distinto, cercano a los anteriores y estratigráficamente por encima de éstos. En general la palinoflora identificada es bastante uniforme en todos los yacimientos; no así la macroflora que presenta peculiaridades en alguno de ellos. Los datos paleobotánicos ponen de manifiesto una vegetación muy diversa con géneros y especies característicos de medios montanos, abanicos aluviales, márgenes lacustre-palustres y lagunares. Los resultados del estudio de la macroflora y de los palinomorfos se representan y ubican en un perfil sintético y en un bloque diagrama esquemático. 
La asociación de vertebrados fósiles está constituida por dos taxones de reptiles (un lacértido y un ánguido), y entre los mamíferos el esciúrido Heteroxerus cf. rubricati, el glírido cf. Armantomys sp., el cricétido Megacricetodon collongensis - crusafonti, un insectívoro y varios restos de lagomorfos no determinables taxonómicamente. Desde un punto de vista bioestratigráfico esta asociación faunística puede situarse dentro del Mioceno Medio en el Aragoniense Superior, entre la zonas F, $\mathrm{G}_{1}$ y $\mathrm{G}_{2}$ de Daams y Freudenthal (1988a) correlacionables con la unidad NM 6 según la biozonación de Mein (1975). El medio ambiente indicado por esta fauna sería de clima relativamente seco y fundamentalmente abierto.

Palabras clave: Paleobotánica, Palinología, macroflora, vertebrados fósiles, Mioceno, cuenca de Madrid.

\section{INTRODUCCIÓN}

En este trabajo se exponen algunos aspectos paleoambientales de la Cuenca miocena de Madrid, hace aproximadamente 15 millones de años, mediante el estudio del registro fósil procedente de seis yacimientos situados en la "Unidad Intermedia" del Mioceno en los que aparecen macrorrestos vegetales, palinomorfos y microvertebrados.

Fernández Marrón et al. (2002) dan a conocer los resultados preliminares del estudio de los vegetales fósiles procedentes de dos yacimientos (Fig.1, yacimientos 1 y 2) descubiertos como consecuencia de la prospección y muestreo paleontológico de las obras de construcción de la Estación Depuradora de Aguas Residuales (EDAR) de la Cuenca media-alta del arroyo Culebro, en el ramal-colector de Parla, dentro del término municipal de Pinto. Se determinaron restos foliares, frutos, pólenes y esporas.

Posteriormente, se han encontrado nuevos yacimientos de plantas fósiles y pisadas de aves en los terrenos del ramal de Fuenlabrada que termina también en la EDAR de la cuenca alta del arroyo Culebro (Fig. 1, yacimientos 3, 4 y 5) así como restos de pequeños vertebrados en el Ramal de Parla, en un paquete de arcillas arenosas verdes oscuras, muy bioturbadas por raices (Fig.1, yacimiento 6).

Los yacimientos se descubrieron como consecuencia de las obras de excavación de la EDAR y sus colectores, por lo que una vez finalizada la instalación de éstos, todos los yacimientos han quedado enterrados y no hay posibilidad de realizar nuevos muestreos.

\section{MARCO GEOLÓGICO}

Los yacimientos se ubican, desde el punto de vista geológico, en la Cuenca del Tajo, dentro de la Cuenca de Madrid, que se corresponde con una amplia depresión de origen tectónico de más de $15.000 \mathrm{~km}^{2}$ con escasos relieves dominados por amplias planicies o páramos.

La Cuenca miocena del Tajo se rellenó mediante el aporte de sedimentos de cursos fluviales procedentes de dos áreas fuentes, una septentrional del Sistema Central y otra meridional de los Montes de Toledo. Estos sistemas fluviales, según disminuían en energía, distribuyeron los materiales por diferencias granulométricas, depositán- dose los gruesos en los bordes de la cuenca, más próximos a las sierras y los finos hacia el centro de ésta. De los depósitos detríticos groseros (conglomerados, arcosas) se pasa a una facies "intermedia de transición", constituida por arenas micáceas y lutitas. Los sedimentos del centro de la cuenca son de origen químico constituidos, por carbonatos y evaporitas que se depositaron en lagos someros (Hoyos et al., 1985).

Los yacimientos estudiados se encuentran en el sector central de la Cuenca de Madrid, dentro de los tramos medios de la sedimentación miocena, en la base de la Unidad litoestratigráfica denominada "Unidad Intermedia", que constituye una banda bastante continua entre los sistemas de abanicos aluviales, de composición eminentemente arcósica, procedentes del Sistema Central y las facies lacustres del centro de la cuenca (Calvo et al., 1989).

\section{ESTRATIGRAFÍA DE LAS SECCIONES ESTUDIADAS}

Los estudios estratigráficos realizados en el área donde se ubican los colectores de la EDAR del arroyo Culebro muestran la presencia de dos tramos bien diferenciados. El inferior, está constituido esencialmente por unas alternancias de lutitas tableadas oscuras, finamente laminadas, con intercalaciones arenosas en las que se observan capas con ripples y cuerpos de arenas finas con biotita dispuestas en niveles muy continuos no canalizados. A techo de este tramo inferior, lutitas masivas con carbonatos vadosos. Las secuencias son grano-crecientes. Entre los planos de estratificación de las lutitas laminadas y tableadas aparecen yesos intercalados y huellas de desecación en los limos. Las lutitas laminadas se depositarían bajo una lámina de agua somera corroborado por la presencia de icnofósiles de pequeños limícolas y anátidas (Fernández Marrón et al., 2002). La lámina de agua sufriría oscilaciones de nivel dando lugar a carbonatos vadosos, llanuras fangosas y apareciendo las típicas huellas de retracción por desecación de la arcilla. Este tramo inferior está representado en las columnas estratigráficas levantadas en el pk. 3,860, en la parte inferior de la de Hinca AVE, en el pk. 4,880 del Ramal de Parla y en las 


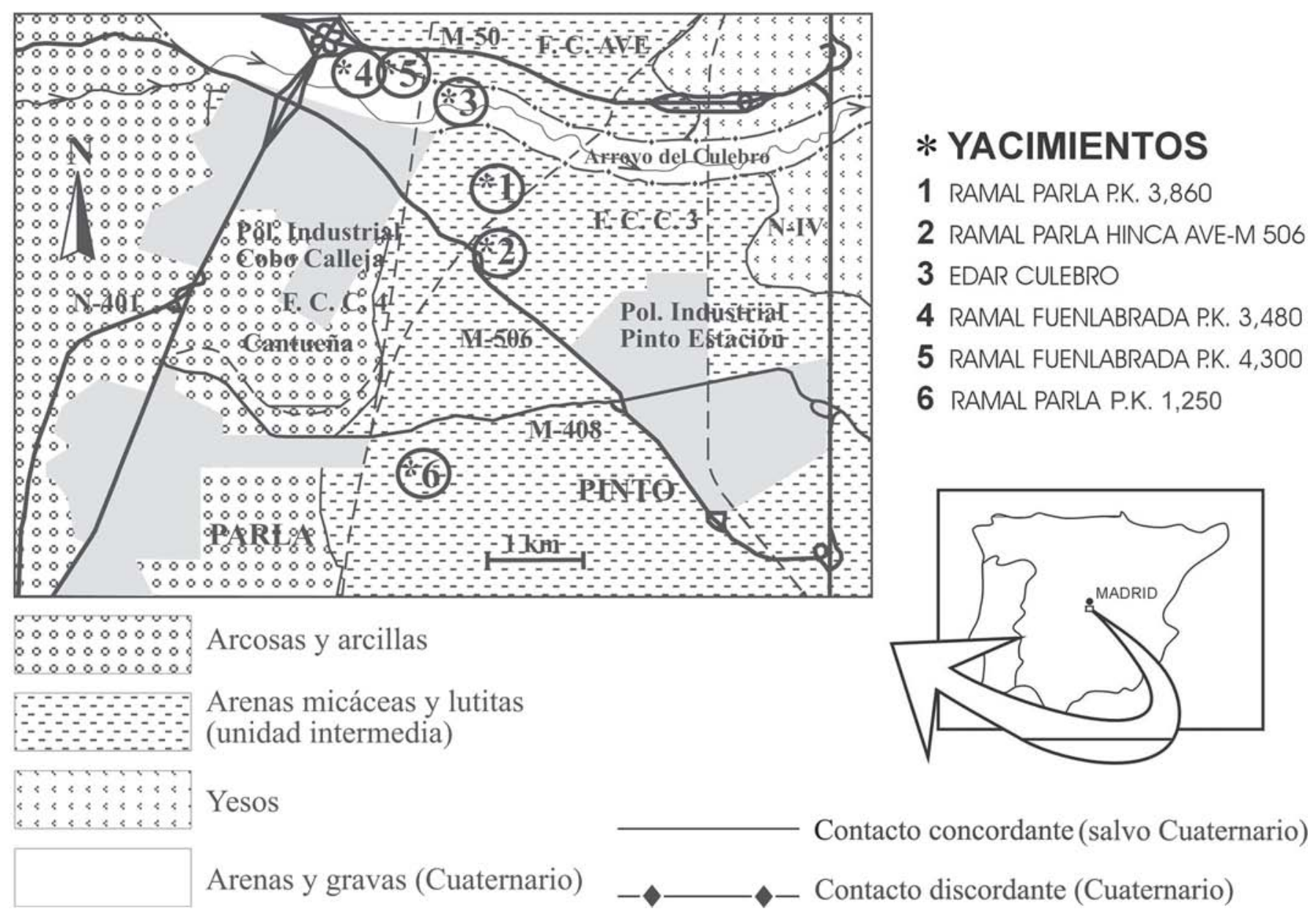

Figura 1. Situación geográfica y geológica de los yacimientos. Geographical and geological location of the studied outcrop.

secciones del pk. 3,480 y pk. 4,300 del Ramal de Fuenlabrada. En estas secciones se ubican respectivamente los yacimientos 1, 2, 3, 4 y 5 (Fig. 1). Las secciones completas donde se han descubierto los yacimientos 1 (pk. 3,860 de la canalización) y 2 (Hinca AVE) ya han sido descritas detalladamente en Fernández Marrón et al.. (2002).

El tramo superior, hacia el sur de la obra, donde se ubica el yacimiento 6 (pk. 1,250 del Ramal de Parla) está constituido por arenas medias y gruesas, bien lavadas. En los niveles canalizados, altamente erosivos, hay cantos de lutita en la base, pasando a carbonatos vadosos con terrígenos. No aparecen yesos intercalados entre los planos de estratificación. Por encima hay un nivel de arcillas arenosas verdes oscuras de aspecto jabonoso y satinado muy bioturbadas por raices, donde se han hallado los restos fósiles de microvertebrados.

Los espesores, litologías, rasgos sedimentarios y contenido paleontológico de cada una de las sucesiones se detallan en las columnas estratigráficas correspondientes así como su correlación efectuada en función de criterios litológicos (Fig. 2).

\section{MÉTODOS}

Para los estudios palinológicos se han tratado $100 \mathrm{gr}$ de sedimento no alterado de cada una de las muestras, dada la pobreza en palinomorfos observada en los ensayos previos.

Las técnicas utilizadas para la extracción de los palinomorfos son las estándares en este tipo de análisis y consisten fundamentalmente en la eliminación de carbonatos, sílice y silicatos mediante ácidos $(\mathrm{ClH}$ y $\mathrm{FH})$, oxidación parcial de la materia orgánica con ácido nítrico y clorato potásico, y eliminación de humatos con álcalis diluidos. Después de cada etapa son necesarios sucesivos lavados, filtrados y centrifugados. El residuo palinológico resultante se engloba y dispersa en glicerogelatina.

Las microfotografias se realizaron con una cámara Wild Photoautomac MPS45 adaptada a un Microscopio óptico Leitz Laborlux.

Los restos fósiles de microvertebrados proceden del lavado y tamizado de $130 \mathrm{~kg}$ de sedimento. Los dientes de micromamíferos se han medido con una lupa dotada de un micrómetro de $0,025 \mathrm{~mm}$ de precisión. Las medi- 


\section{Especies representadas por tipo de habitat}
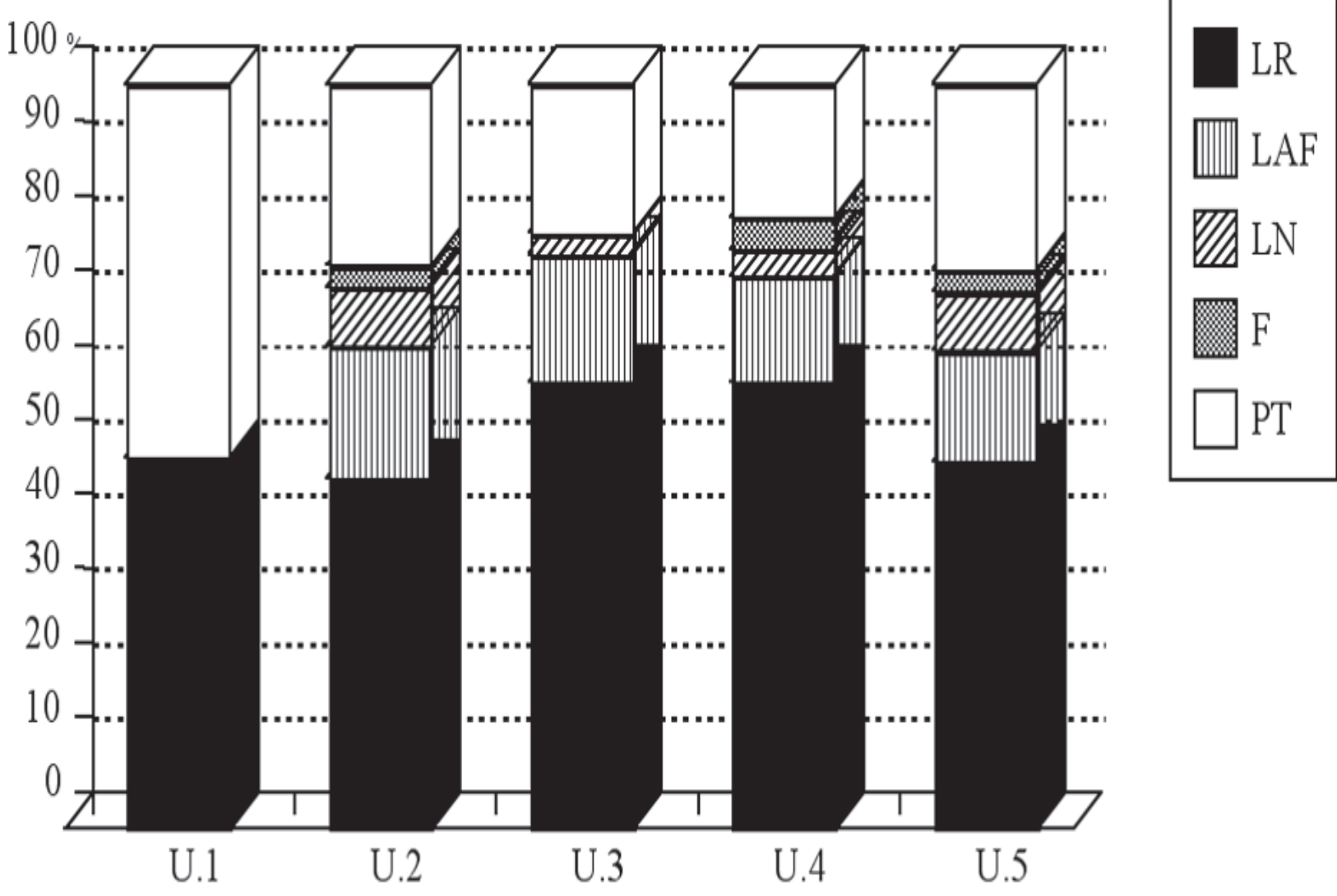

Figura 2. Columnas estratigráficas y sus correlaciones.

Stratigraphic sections and their correlations

das se expresan en milímetros y corresponden a la longitud (L) y anchura (A) máximas de la superficie oclusal. Los dibujos se han realizado con una cámara clara acoplada a una lupa binocular Nikon SMZ-10.

Los restos de macroflora y microvertebrados fósiles están depositados en el Museo Nacional de Ciencias Naturales de Madrid. La macroflora está siglada como PD (Parla Depuradora, correspondiente al yacimiento 1), PR (Parla Ramal, yacimiento 2) TM (Toledo-Madrid, yacimiento 4) y MAT (Matadero, Yacimiento 5). Los microvertebrados están registrados bajo la denominación de "Parla Ramal", con las siglas de abreviatura "PRM", en la caja ${ }^{\circ} 37$ con la numeración del 1 al 30 .

\section{LOS VEGETALES FÓSILES}

La flora de los yacimientos 1 y 2 , que fueron los primeros que se descubrieron al realizar las obras de la canalización, ya fue listada en Fernández Marrón et al. (2002). Posteriores hallazgos proporcionaron más restos foliares, con peor (yacimiento 3) o mejor conservación (yacimientos 4 y 5) que los estudiados previamente, pero que prácticamente correspondían a las mismas especies.

El estudio de la flora fósil pone de manifiesto la presencia de una vegetación muy diversa, con especies características de ambientes muy variados: terrestres, ripícolas y acuáticos. En las Tablas 1 y 2 se relacionan sistemáticamente los diferentes taxones de macroflora y palinomorfos determinados en los yacimientos 


\section{CONSIDERACIONES SOBRE LOS VEGETALES FÓSILES Y SU DISTRIBUCIÓN PALEOECOLÓGICA}

Entre los restos de macroflora hallados destaca la abundancia de pequeños foliolos, junto con hojas de tamaño mediano (microfilas). Algunas de estas hojas simples están recubiertas de una película ferruginosa que favorece su conservación y permite una observación más precisa de la nerviación, el margen, etc. Otras, en cambio, se presentan recubiertas por una película arcillosa que le da un aspecto jabonoso e impide observar con nitidez los ras- gos morfológicos. Por lo general las hojas de mayor tamaño corresponden a impresiones y parecen estar relacionadas con corrientes activas de agua, mientras que restos foliares pequeños y ligeros como los foliolos de las leguminosas y de hojas alargadas y estrechas, mas fragmentadas, pudieron ser transportadas por el viento a un ambiente más lagunar. Así mismo han aparecido un número considerable de diásporas como frutos y semillas, sobre todo aladas, cuyo transporte al área de sedimentación pudo ser también aéreo. Esta selección ligada al transporte de los macrorrestos pudo generar asociaciones que deformen la composición de la flora original (Martín Closas y Picó, 1998).

\section{TABLA 1}

\author{
Gymnospermophyta \\ Orden Coniferales \\ Fam. Pinaceae \\ Pinus sp. \\ Picea sp. \\ Semilla alada de Pinaceae (Fig. 3f) \\ Fam. Taxodiaceae \\ Glyptostrobus europaeus (Brongniart 1833) Unger 1850 \\ (Fig. 3f*) \\ Fam. Cupresaceae \\ Tetraclinis salicornioides (Unger 1841) Kvacek1986
}

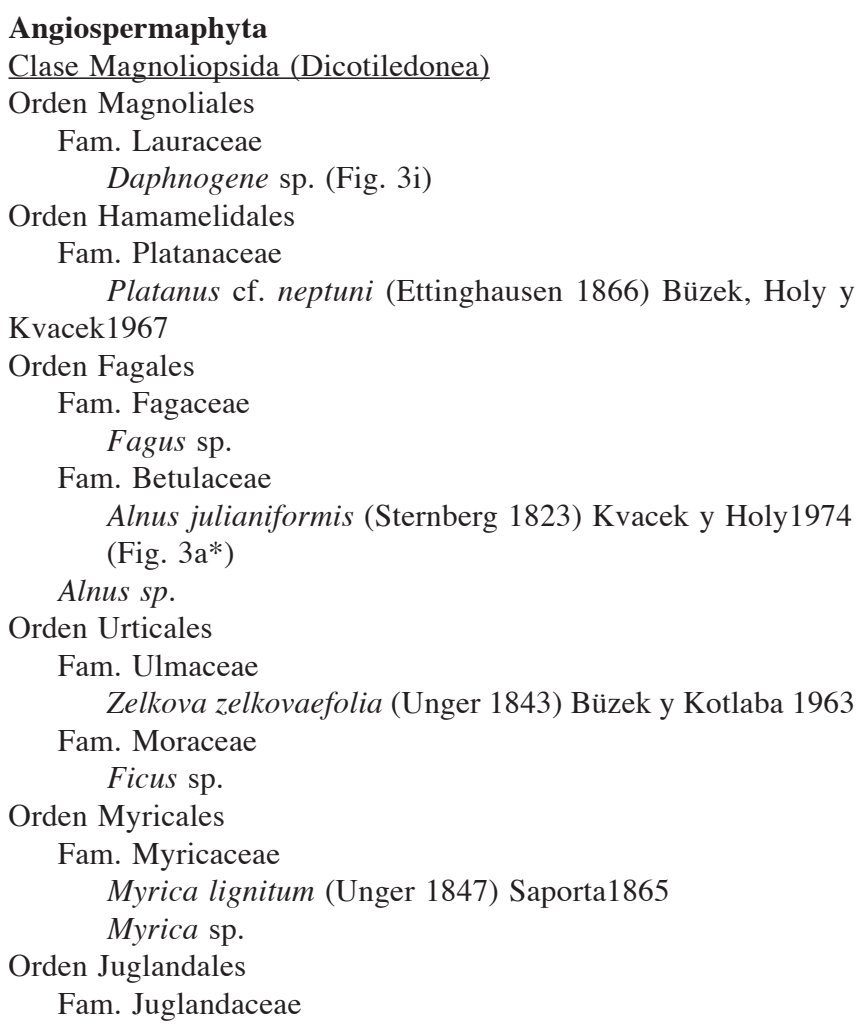

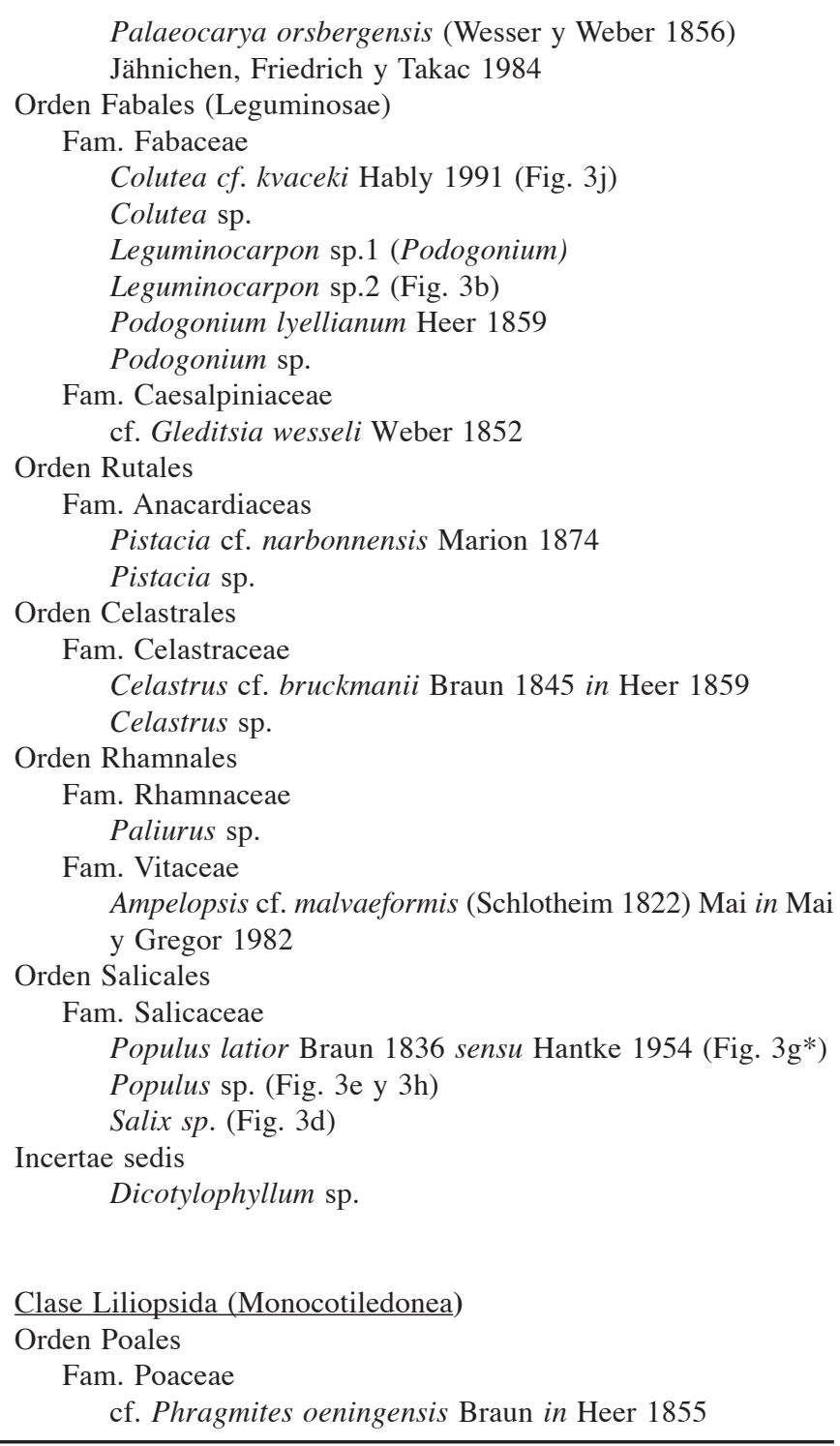

Tabla 1. Relación de taxones de la macroflora determinada indicando los representados en las figuras. Las ilustraciones marcadas con un asterisco (*) han sido previamente publicadas en Fernández Marrón et al. (2002).

List of macroflora taxa determinated showing the figurated. The pictures indicate with a asterisk (*) has been published in Fernández Marrón et al. (2002). 
TABLA 2

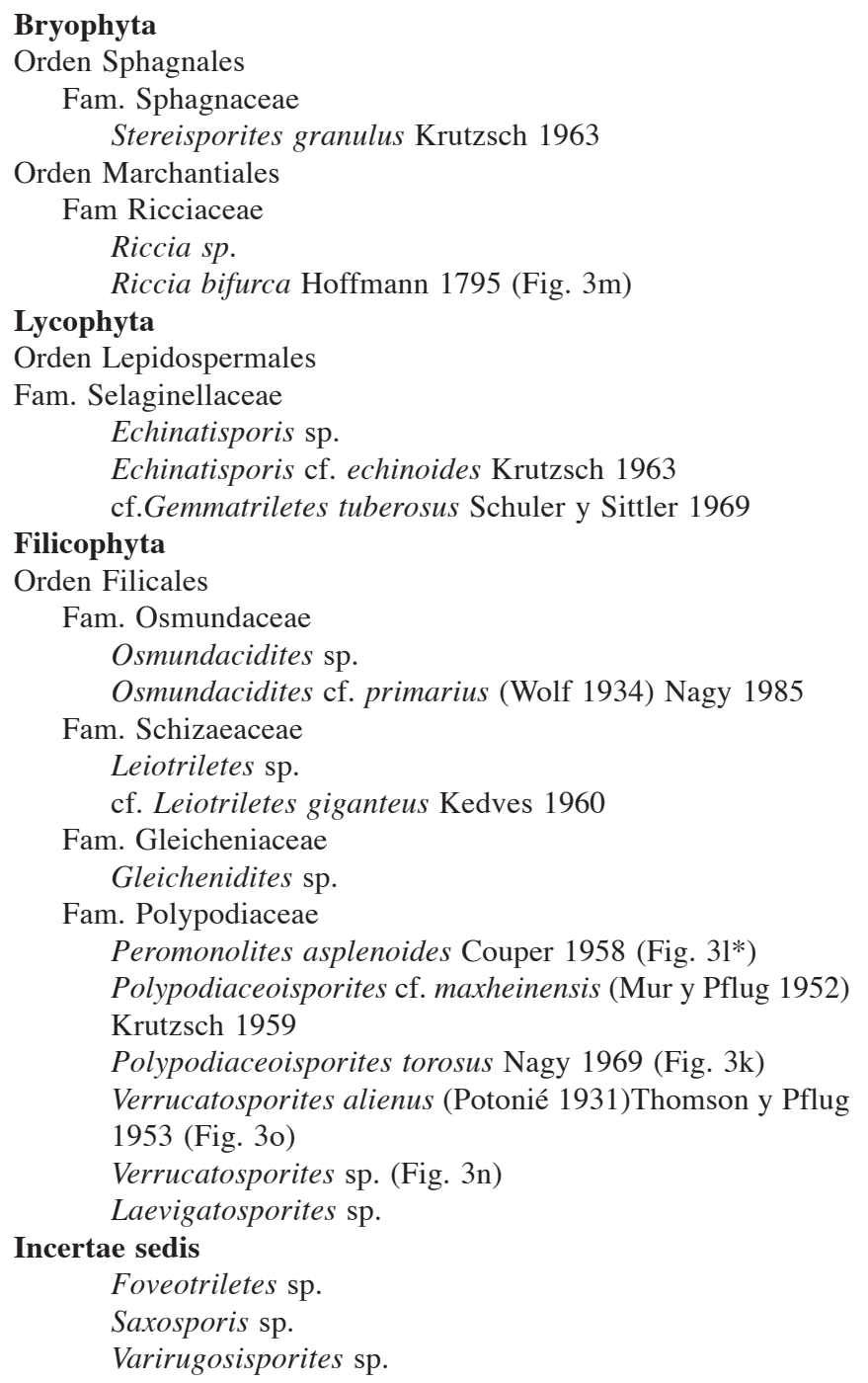

Gymnospermophyta

Orden Cycadales

Fam. Cycadaceae

Cycadopites sp.

Orden Gnetales (Ephedrales)

Fam. Ephedraceae

Ephedripites sp.

Orden Coniferales

Fam. Pinaceae

Pityosporites absolutus (Thiergart 1938) Pflug y Thomson 1953

Pityosporites alatus (Potonié 1931) Thomson y Pflug 1953 Pityosporites labdacus (Potonié 1931) Thomson y Pflug 1953 (Fig. 4a)

Pityosporites microalatus (Potonié 1931) Thomson y Pflug 1953 (Fig. 4b-4c)

Pityosporites sp.1

Pityosporites sp.2 (cf. Keteleeria $\mathrm{sp}$. )

Cf. Pityosporites libellus Potonié 1931

Tsugaepollenites viridifluminipites (Wodehouse 1933)

Potonié 1958.

Tsugaepollenites sp.

Fam. Podocarpaceae

Podocarpidites podocarpoides (Thiergart 1958) Krutzsch
1971 (Fig. 4e*)

Podocarpidites sp.

Fam. Araucariaceae Araucariacites australis Cookson 1947 Araucariacites sp. (Fig. 4d)

Fam. Taxodiaceae Sciadopitypollenites sp.

\section{Angiospermaphyta}

Clase Magnoliopsida (Dicotiledonea)

Orden Magnoliales

Fam. Magnoliaceae Magnoliopollis sp. (Fig. 4j)

Orden Hamamelidales

Fam. Hamamelidaceae

Periporopollenites stigmosus (Potonié 1931) Pflug y Thomson 1951

Orden Fagales

Fam. Fagaceae

Tricolporopollenites genuinus (Potonié 1934) Thomson y Pflug 1953 (Fig. 4g*)

Fam. Betulaceae

Polyvestibulopollenites verus (Potonié 1931) Thomson y Pflug 1953

Orden Urticales

Fam. Ulmaceae

Polyporopollenites verrucatus Thiele- Pfeiffer 1980

Polyporopollenites $\mathrm{cf}$. undulosus (Wolff 1934) Thomson y Pflug 1953

Cf. Polyporopollenites sp.

Zelkovaepollenites potoniei Nagy 1969

Orden Juglandales

Fam. Juglandaceae

Caryapollenites sp.

Orden Myrtales

Fam. Onagraceae

Onagraceaepollenites sp.

Orden Celastrales

Fam. Aquifoliaceae Ilexpollenites iliacus (Potonié 1931) Thiergart 1937

Orden Salicales

Fam. Salicaceae Inaperturopollenites incertus Thomson y Pflug 1953(Populus sp.?) (Fig. 4f)

Orden Malvales

Fam. Malvaceae Malvaceaepollenites sp. (Fig. 4h*)

Orden Ericales

Fam. Ericaeae

Ericipites longisulcatus Wodehouse 1933

Orden Centrospermales

Fam. Chenopodiaceae Chenopodipollis sp.

Fam. Chenopodiaceae/Caryophyllaceae/ Alismataceae Orapollis postdamensis Krutzsch 1966

Cf. Orapollis sp.

Orden Poligonales

Fam. Polygonaceae

Persicarioipollenites crassicus Krutzsch 1962

Persicarioipollenites cf. minor Krutzsch 1962

Orden Asterales

Fam. Compositae (Asteraceae) 


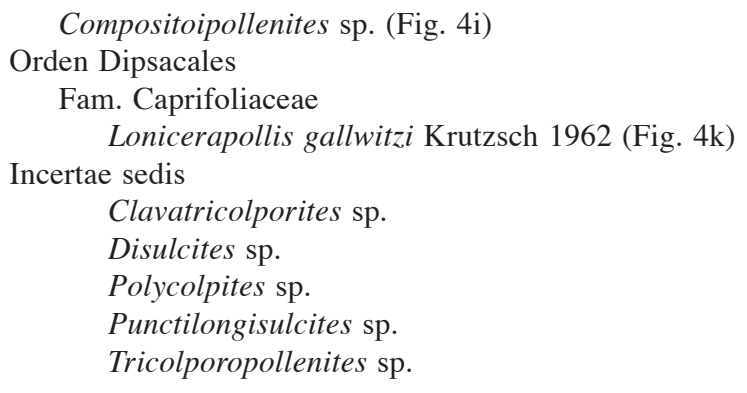

Clase Liliopsida (Monocotiledonea)

Orden Poales

Fam. Poaceae

Graminidites sp. (Fig. 4n)

Orden Arecales
Fam. Arecaceae

Arecipites convexus (Thiergart 1937) Krutzsch 1970

Arecipites eopapillosus Krutzsch y Vanhoorne 1977

(Fig. 4o)

Arecipites cf. monocolpatus (Doktorowicz-Hrebnicka 1957)

Krutzsch 1970

Arecipites sp.

Sabalpollenites cf. areolatus (Potonié 1934) Potonié 1958

(Fig. 4m)

cf. Sabalpollenites sp.

Orden Typhales

Fam. Sparganiaceae/Thyphaceae

Aglaoreidia cyclos (Erdtman 1960) Fowler 1971

Fitoclastos

Traqueidas (Fig. 4l)

Cutículas

Tabla 2. Relación de palinomorfos determinados con indicación de los representados en las figuras. Las ilustraciones marcadas con un asterisco (*) han sido previamente publicadas en Fernández Marrón et al. (2002).

List of palynomorphs determinated showing the figurated. The pictures indicate with a asterisk (*) has been published in Fernández Marrón et al. (2002).

Mientras las asociaciones esporopolínicas determinadas en los cinco yacimientos son similares en su composición, la macroflora presenta peculiaridades en los distintos yacimientos. En concreto, en el yacimiento 3 la macroflora encontrada no es identificable y el yacimiento 4 presenta una asociación foliar muy monótona constituida casi exclusivamente por hojas simples, de borde dentado, pertenecientes a los géneros Salix y Populus que serían objeto de transporte hidrófilo. El yacimiento 2 presenta numerosos y variados macrorrestos correspondientes generalmente a foliolos (Colutea, Gleditsia, Podogonium) y frutos de leguminosas (Leguminocarpon), hojas de Myrica, semillas aladas de Pináceas, etc., lo que parece indicar una zona de depósito de elementos transportados por el viento. Estas deducciones, se han efectuado sobre elementos tafonómicos (Fernández López, 2000) y son las inherentes a las características de los ejemplares (distribución por tamaños, conservación, alteraciones, etc)

En el yacimiento 1 se han identificado macrorrestos y palinomorfos propios de bosques húmedos como Glyptostrobus, Myrica, etc, lianas como Ampelopsis y elementos de bosques mesofíticos caducifolios como Fagus, Alnus, Populu, Zelkova, Gleditsia y perennifolios Tsuga, Abies, Pinus, Tetraclinis y Sciadopitys (Bernor et al., 1988; Hably y Kvacek, 1998).

Los palinomorfos muestran el carácter heterogéneo de la asociación con elementos acuáticos Riccia, Esparganiáceas, Tifáceas, etc, junto con formas polínicas ligadas a la humedad atribuibles a Onagráceas, Alnus, Populus, Carya, etc, y otras de ambiente seco como Ericáceas, Compuestas, etc (Pais, 1981; Fernández Marrón et al., 2002). Así mismo están representados gran cantidad de elementos adémicos (Fernández López, 2000) ya que entre las formas polínicas destacan por su abundancia las atribuidas a Coníferas de dispersión anemófila y que probablemente provenían de áreas montanas más o menos alejadas de la de depósito.

El análisis del contenido de las asociaciones registradas de macrorrestos vegetales y palinomorfos, permite establecer una distribución de la flora y representarla en un bloque diagrama esquemático (Fig. 5) basado en Lomoschitz et al.(1985), Calvo (2000) y Sánchez et al. (2000). También se figuran los componentes de aquella en un perfil elaborado de acuerdo con el citado bloque diagrama, donde se ponen de manifiesto los distintos ambientes reconocidos con su vegetación característica (Fig. 6).

La vegetación fósil registrada en la "Unidad Intermedia" del Mioceno del Sur de la Comunidad de Madrid correspondería a distintos ambientes paleoecológicos. Las agrupaciones floristicas se han establecido siguiendo criterios actualísticos.

Los bosques de Pináceas, Tsuga, Fagus, Zelkova e Ilex poblarían las cumbres y laderas montañosas. Los ambientes de abanico aluvial se caracterizarían por una vegetación adaptada a la xericidad en praderas abiertas, donde se desarrollaban matorrales y arbustos de distintos géneros y familias: Podogonium, Caesalpináceas, Pistacia, Robinia, Colutea, Paliurus, Ephedripites, Celastrus, Gramíneas, Compuestas y Ericáceas.

La zona entre el abanico aluvial y el margen lacustrepalustre se caracterizaría por un ambiente ripícola, con presencia de praderas húmedas y bosques de ribera con Populus, Alnus, Carya y Daphnogene. Serían zonas de inundación por desbordamiento de los ríos, por aumento de los límites lacustres o por aguas aflorantes al pie de los abanicos.

La presencia de Riccia, Taxodium, etc sugiere la existencia de zonas palustres. En los deltas y márgenes 

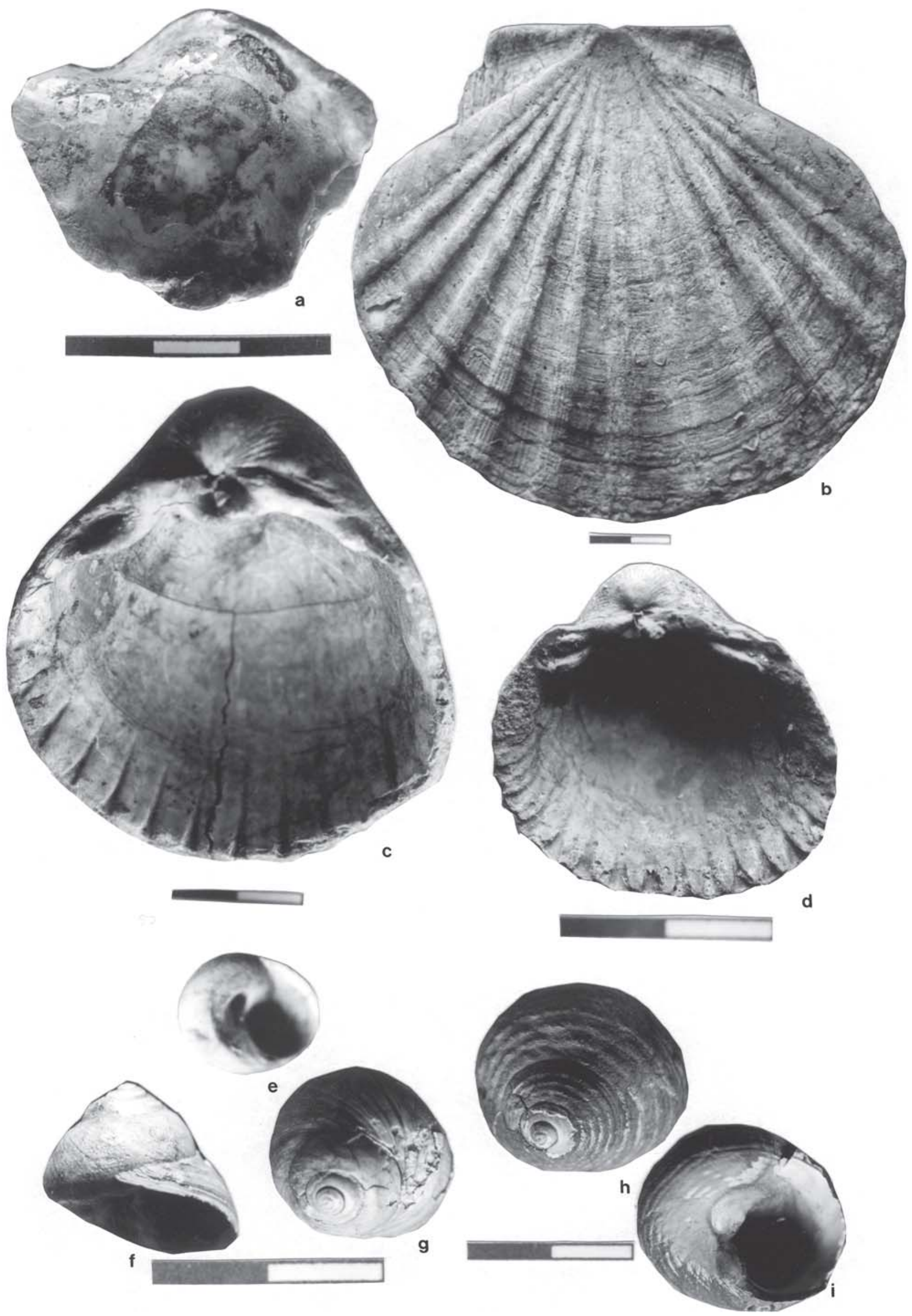
lacustres crecerían Myrica, Phragmites, Esparganiáceas, Tifáceas, Glyptostrobus y algas de agua dulce.

La macroflora presenta, en general hojas de tamaño reducido y consistencia coriácea lo que parece indicar aridez, excepto en el yacimiento 4 que está constituida casi exclusivamente por hojas de Salicáceas y que podría corresponder a una zona de ambiente ribereño en el área interpretada. Entre las formas polínicas destacan por su abundancia las Pináceas, normal dada la gran cantidad de granos de polen que producen estas plantas y su amplia dispersión, pero el hecho de que también estén presentes restos de conos y semillas aladas indica una presencia significativa de estas Coníferas. La abundancia y diversidad de pinos es característica de climas continentales de semiáridos a áridos, de escasa precipitación y frecuente sequedad ambiental (Ceballos y Ruiz de la Torre, 1971).

De la vegetación determinada, características de los sedimentos y distribución de ambientes sedimentarios (Calvo, 2000) se puede deducir la existencia en la "Unidad Intermedia" del Mioceno de Madrid de un clima semiárido, con oscilaciones estacionales: una estación cálida, más o menos húmeda y otra cálida y seca.

\section{LOS VERTEBRADOS FÓSILES}

Los restos de vertebrados encontrados en el yacimiento 6 (Ramal Parla) están listados en la tabla 3. En este apartado se realiza la descripción del material de micromamíferos que ha permitido su identificación taxonómica.

CLASE MAMMALIA

ORDEN RODENTIA Bodwich, 1821

Familia Sciuridae Gray, 1821

Género Heteroxerus Stehlin y Schaub, 1951

\section{Heteroxerus cf. rubricati Crusafont, Villalta y Truyols, 1955}

Fig. 7 a

Material y medidas: Un fragmento de $\mathrm{P} / \mathrm{M}$ inferior izquierdo (PRM-6); un $\mathrm{M}_{1-2}$ izquierdo (PRM-7) con $\mathrm{L}=1,69$ y A = 1,76 mm.
TABLA 3

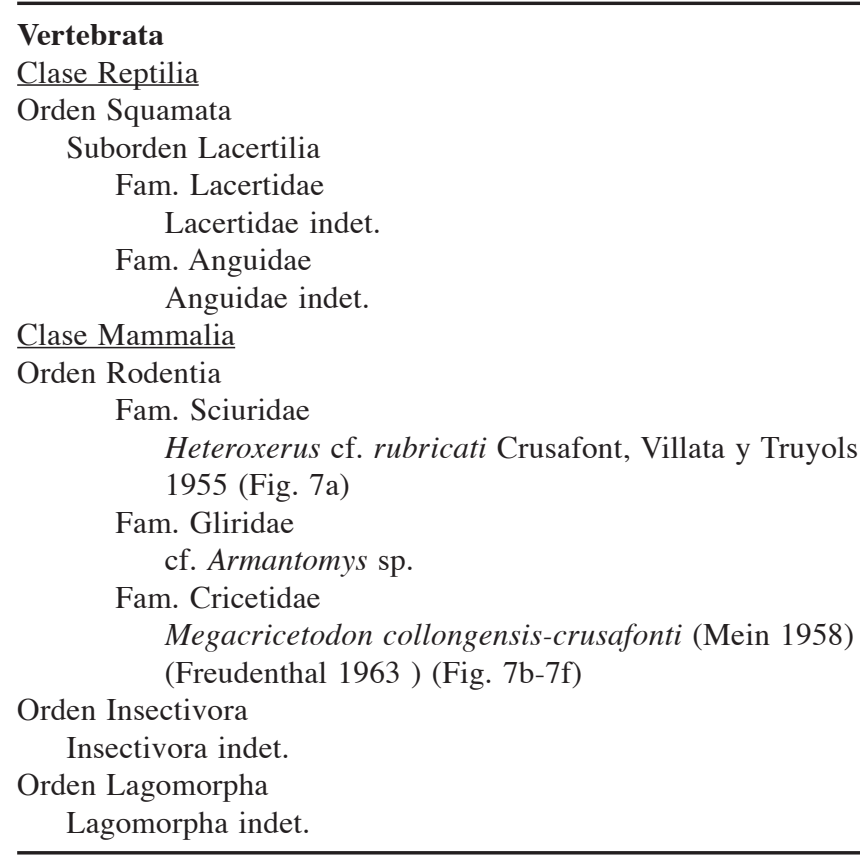

Tabla 3. Relación de la fauna de vertebrados determinada. List of vertebrate fauna determinated.

Descripción y discusión: Los dos dientes están fragmentados y muy erosionados. Su morfología es la característica del género Heteroxerus. Su talla es relativamente pequeña, se encuentra dentro de la variabilidad de la de las diversas poblaciones de $H$. rubricati y es más pequeña que la de la especie H. grivensis (Forsyth Mayor, 1909) (ver: De Bruijn, 1967; Sesé, 1980; Cuenca, 1988).

Familia Gliridae Thomas, 1897

Género Armantomys De Bruijn, 1966

\section{cf. Armantomys sp.}

Material: Fragmento de molar.

Descripción y discusión: A pesar del mal estado de preservación de este fragmento de molar del que sólo se con-

Figura 3. a, Alnus julianiformis (Stenrberg 1823) Kvacek y Holy1974 (x 1); b, Leguminocarpon sp. (x2); c, Glyptostrobus europaeus (Brongniart 1833) Unger 1850 (x2); d, Salix sp. (x2); e, Populus sp. (x1); f, Semilla alada de Pinaceae (x2); g, Populus latior Braun 1836 sensu Hantke 1954 (x1); h, Populus sp. (x1); i, Daphnogene sp. (x2); j, Colutea cf. kvaceki Hably 1991 (x2); k, Polypodiaceoisporites torosus Nagy 1969 (x500); 1, Peromonolites asplenoides Couper 1958 (x 500); m, Riccia bifurcata Hoffman 1795 (x1000); n, Verrucatosporites sp. (x 1000); o, Verrucatosporites alienus (Potonié 1931) Thomson y Pflug 1953 (x 1000).

$\boldsymbol{a}$, Alnus julianiformis (Stenrberg 1823) Kvacek and Holy1974 (x 1); b, Leguminocarpon sp. (x2); c, Glyptostrobus europaeus (Brongniart 1833) Unger $1850(x 2) ; \boldsymbol{d}$, Salix $s p .(x 2) ; \boldsymbol{e}$, Populus $s p .(x 1) ; \boldsymbol{f}$, Wing seed of Pinaceae (X2); $\boldsymbol{g}$, Populus latior Braun 1836 sensu Hantke 1954. (xl); h, Populus $s p .(x 1)$; $\boldsymbol{i}$, Daphnogene $s p .(x 2)$; $\boldsymbol{j}$, Colutea $c f$. kvaceki Hably 1991 (x2); $\boldsymbol{k}$, Polypodiaceoisporites torosus Nagy 1969 (x500); $\boldsymbol{l}$, Peromonolites asplenoides Couper 1958 (x 500); $\boldsymbol{m}$, Riccia bifurcata Hoffman 1795 (x1000); $\boldsymbol{n}$, Verrucatosporites sp. (x 1000); o, Verrucatosporites alienus (Potonié 1931) Thomson and Pflug 1953(x 1000). 

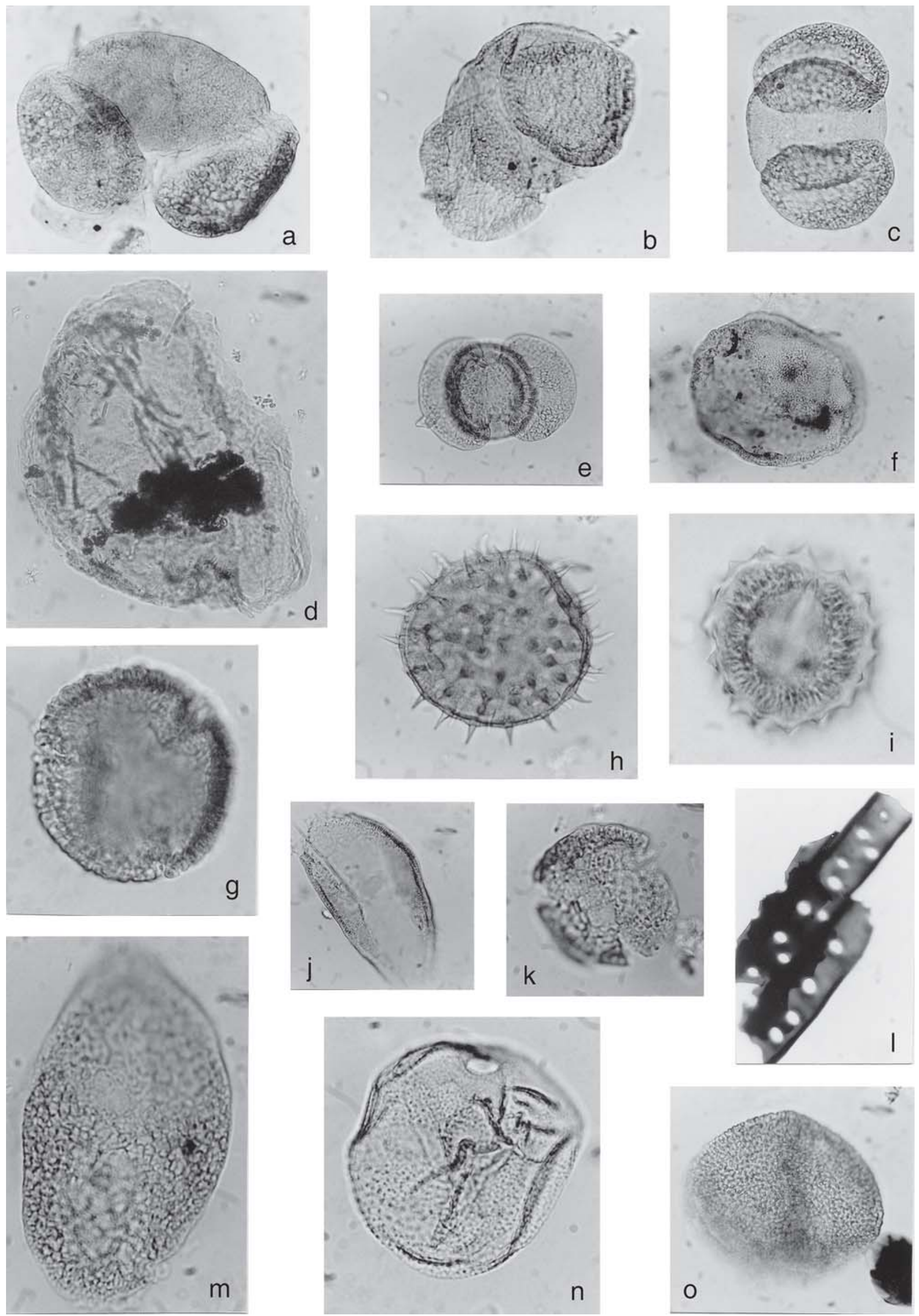

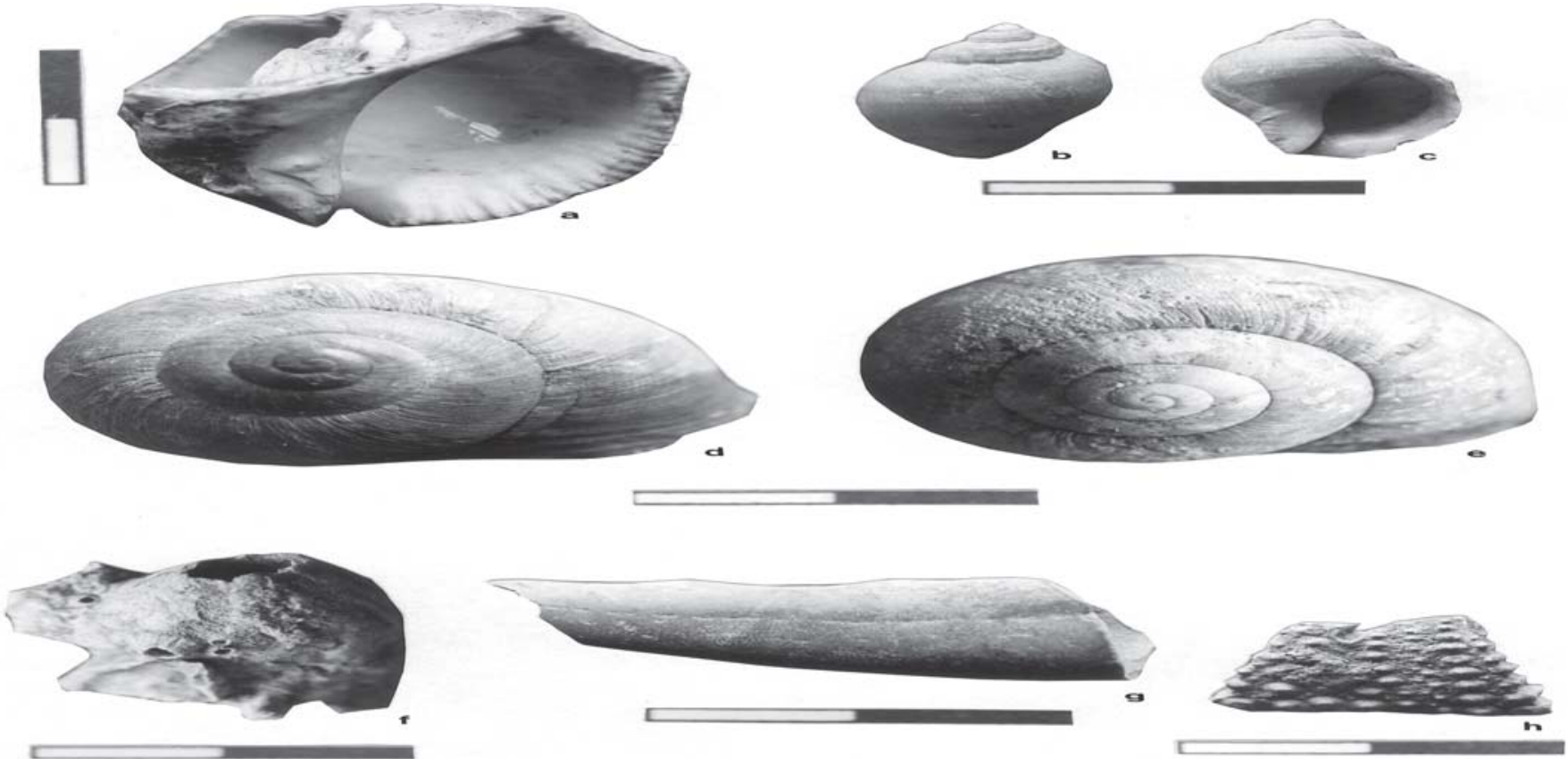

Figura 5. Bloque diagrama esquemático basado en Lomoschitz et al.(1985), Calvo (2000) y Sánchez et al. (2000) con su vegetación característica.

Block diagram after Lomoschitz et al.(1985), Calvo (2000) and Sánchez et al. (2000) with the characteristic vegetation.

servan las dos crestas posteriores, la forma curva tan característica de éstas y la hipsodoncia del diente son las propias del género Armantomys.

Familia Cricetidae Stehlin y Schaub, 1951

Género Megacricetodon Fahlbusch, 1964

\section{Megacricetodon collongensis - crusafonti (Mein, 1958) - (Freudenthal, 1963 ) Fig. 7 b-f}

Material: Un $M_{1}$; un $M_{2}$; dos $M^{1}$; tres $M^{2} y$ tres $M^{3}$. Sus medidas están en la Tabla 4.
Descripción y discusión: La talla de esta población es mayor que la de $M$. primitivus (Freudenthal, 1963) y, en general, también mayor que la de $M$. collongensis es sólo comparable a los valores máximos de algunas poblaciones de esta última especie, y entra sin embargo dentro de la variabilidad de las diversas poblaciones de Megacricetodon collongensis - crusafonti y de M. crusafonti descritas por Daams y Freudenthal (1988b).

Tanto M. collongensis como M. crusafonti están muy bien representadas en numerosos yacimientos de la Cuenca de Madrid. Con respecto a dichas poblaciones se puede señalar que el material de Parla es de mayor talla en general que el de $M$. collongesis de los yacimientos de Moratines (Alberdi et al., 1981), Henares 2, O’Donell, Cantera del Trapero, San Isidro, Ciudad Pegaso y Almo-

Figura 4. a, Pityosporites labdacus (Potonié 1931) Thomson y Pflug1953 (x 500); b y c, Pityosporites microalatus (Potonié 1931) Thomson y Pflug1953 (x 500); d, Araucariacites sp. (x 1000); e, Podocarpidites podocarpoides (Thiergart 1958) Krutzsch 1971 (x 500); f, Inaperturopollenites incertus Thomson y Pflug 1953 (x 500); g, Tricolporopollenites genuinus (Potonié 1934) Thomson y Pfug 1953 (x 1000); h, Malvaceaepollenites sp. (x 500); i,Compositoipollenites sp. (x1000); j, Magnoliopollis sp. (x500); k, Lonicerapollis gallwitzi Krutzsch 1962 (x 500); 1, Traqueida (x300); m, Sabalpollenites cf. areolatus (Potonié 1934) Potonié 1958 (x 1000); n, Graminidites sp. (x1000); o, Arecipites eopapillosus Krutzsch y Vanhoorne 1977 (x 500).

$\boldsymbol{a}$, Pityosporites labdacus (Potonié 1931) Thomson and Pflug1953 (x 500); $\boldsymbol{b}$ and $\boldsymbol{c}$, Pityosporites microalatus (Potonié 1931) Thomson and Pflug 1953 (x 500); d, Araucariacites sp. (x1000); e, Podocarpidites podocarpoides (Thiergart 1958) Krutzsch 1971 (x 500); $f$, Inaperturopollenites incertus Thomson and Pflug 1953 (x 500); g, Tricolporopollenites genuinus (Potonié 1934) Thomson and Pfug 1953 (x 1000); $\boldsymbol{h}$, Malvaceaepollenites sp. (x 500); i,Compositoipollenites sp. (x1000); j, Magnoliopollis sp. (x500); $\boldsymbol{k}$, Lonicerapollis gallwitzi Krutzsch 1962 (x 500); $\boldsymbol{l}$, Wood (X3000); $\boldsymbol{m}$, Sabalpollenites $c f$. areolatus (Potonié 1934) Potonié 1958 (x 1000); n, Graminidites sp. (x1000); o, Arecipites eopapillosus Krutzsch and Vanhoorne 1977 (x 500). 
TABLA 4

\begin{tabular}{lccc}
\hline Molar & N. $^{\circ}$ Catálogo & Longitud & Anchura \\
$M_{1}$ & PRM-8 & 1,64 & 1,02 \\
$M_{2}$ & PRM-21 & - & 0,88 \\
$M^{1}$ & PRM-1 & 1,72 & 1,05 \\
$M^{2}$ & PRM-3 & 1,19 & 1,02 \\
$M^{2}$ & PRM-24 & 1,13 & 0,95 \\
$M^{2}$ & PRM-26 & - & $\pm 0,99$ \\
$M^{3}$ & PRM-4 & 0,88 & 0,83 \\
$M^{3}$ & PRM-25 & 0,86 & 0,90 \\
$M^{3}$ & PRM-5 & 0,74 & 0,79 \\
\hline
\end{tabular}

Tabla 4. Medidas de los ejemplares de Megacricetodon collongensis-crusafonti.

Measurements of the Megacricetodon collongensiscrusafonti specimens.

dóvar (Sesé et al., 1985; López et al., 1987), Somosaguas (López et al., 2000), y sin embargo entra dentro de la variación de la talla de $M$. crusafonti de Paracuellos 3 y 5, y Henares 1 (Sesé et al., 1985).

Aunque la población de Parla es muy escasa, se pueden realizar las siguientes precisiones: su morfología presenta caracteres que le aproximan tanto a $M$. collongensis como a $M$. crusafonti: división del anterocono en el $\mathrm{M}^{1}$, anterocónido sin dividir en el $\mathrm{M}_{1}$ según se aprecia en el único ejemplar disponible a pesar de estar desgastado, mesolofos y mesolófidos relativamente largos o cortos.
ORDEN INSECTIVORA Bodwich, 1821

INSECTIVORA indet.

Material: Un fragmento de diente.

Descripción y discusión: El fragmento de diente parece corresponder posiblemente a un premolar del que se conserva una de las dos raíces y parte (la mitad en altura) de la cúspide principal, que es única y aguda. Dada la fragmentariedad del material no es posible realizar mayores precisiones taxonómicas.

ORDEN LAGOMORPHA Brandt, 1855

LAGOMORPHA indet:

Material: 1 fragmento de $\mathrm{D}_{3}$ y 2 fragmentos de P/M muy desgastados.

Descripción y discusión: El material está en muy mal estado de conservación y no permite hacer ninguna atribución taxonómica precisa.

\section{CONSIDERACIONES SISTEMÁTICAS, BIOESTRATIGRÁFICAS Y PALEOAMBIENTALES DEDUCIDAS A PARTIR DE LOS MICROVERTEBRADOS}

Desde que se describió la primera asociación faunística de micromamíferos de Moratines hallada en la Cuenca de

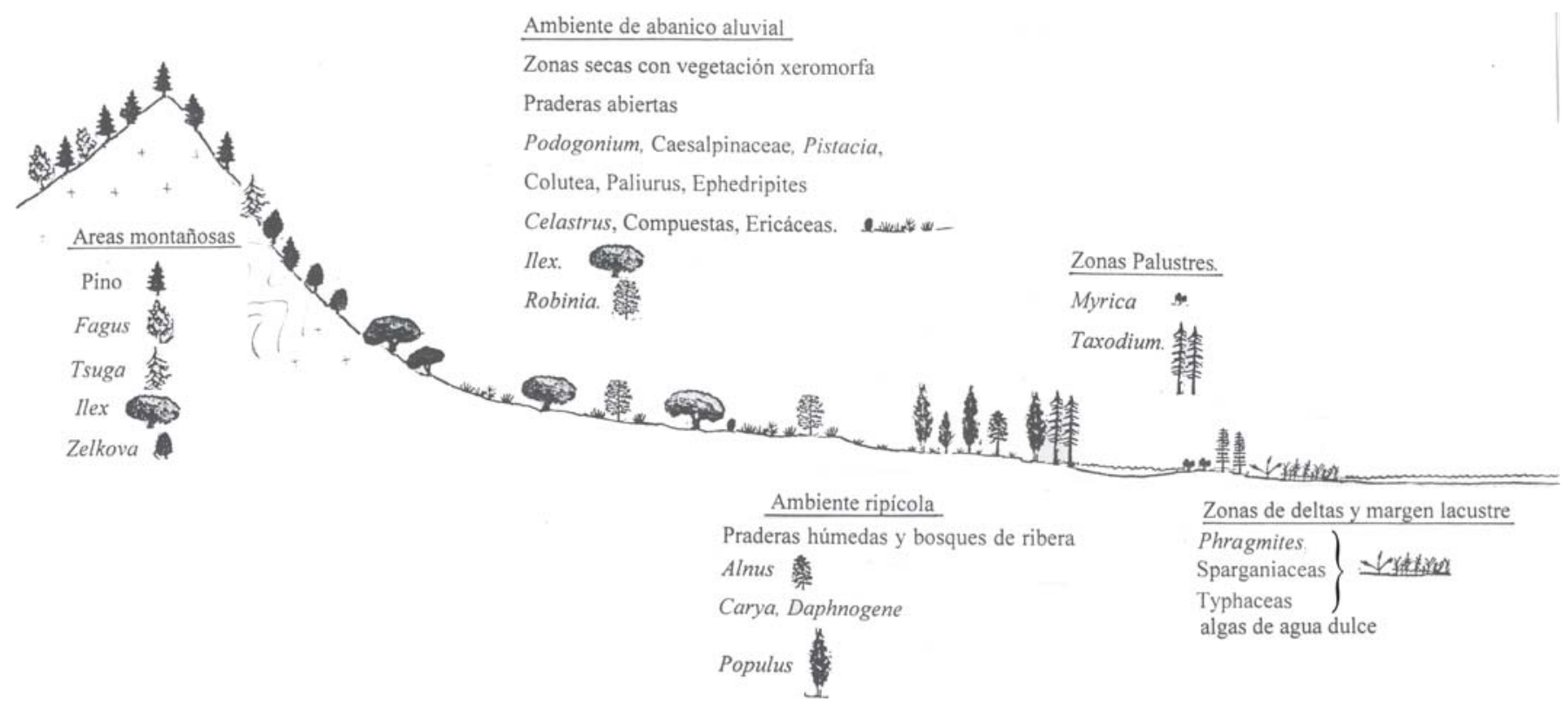

Figura 6. Representación en un perfil esquemático de los distintos paleoambientes y sus paleoasociaciones vegetales determinados. Schematic palaeoenviromental characterization and palaeophytoassociations identified. 

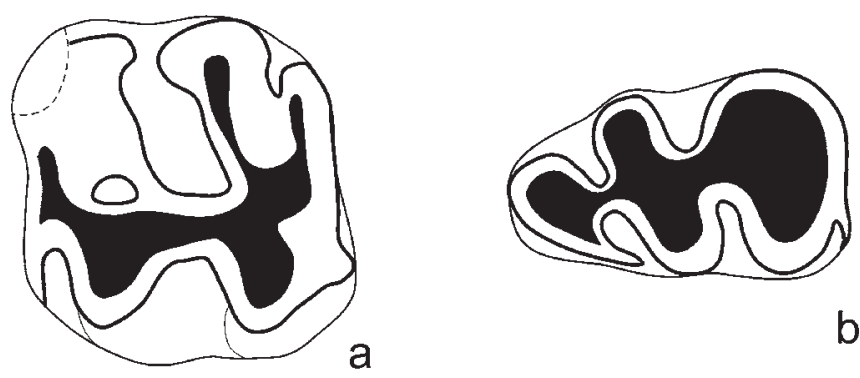

a
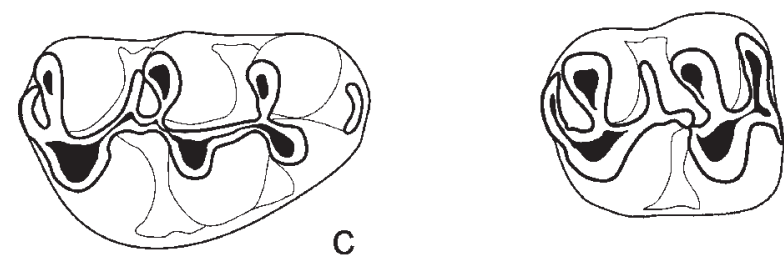

$\mathrm{c}$

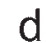

$\mathrm{e}$

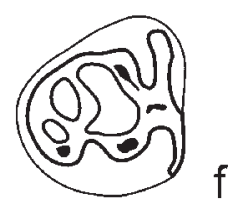

b

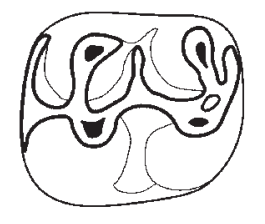

e

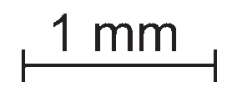

Figura 7. Heteroxerus cf. rubricati Crusafont, Villalta y Truyols 1955 de Parla: a, $\mathrm{M}_{1-2}$ izquierdo (PRM-1). Megacricetodon collongensis-crusafonti (Mein1958)(Freudental 1963) de Parla: b, $\mathbf{M}_{1}$ izquierdo (PRM8); c, $\mathrm{M}^{1}$ dererecho (PRM-1); d, $\mathrm{M}^{2}$ dererecho (PRM-3); e, $\mathrm{M}^{2}$ izquierdo (PRM-24); f, $\mathrm{M}^{3}$ dererecho (PRM-25).

Heteroxerus $c f$. rubricati Crusafont, Villalta and Truyols 1955 from Parla: a, $M_{1-2}$ left (PRM-1). Megacricetodon collongensis-crusafonti (Mein1958)(Freudental 1963) from Parla: $\boldsymbol{b}, M_{1}$ left (PTM-8); c, $M^{1}$ right (PRM-1); d, $M^{2}$ right (PRM-3); e, $M^{2}$ left (PRM-24); $f, M^{3}$ right (PRM-25).

Madrid y atribuida, dentro del Mioceno Medio, al Aragoniense Medio (Alberdi et al., 1981), son muy numerosos los yacimientos de micromamíferos que se han encontrado en dicha cuenca y que constituyen una sucesión de faunas del Aragoniense Medio y Superior, desde la biozona D hasta la G de Daams y Freudenthal (1988 a) (Sesé et al., 1985; López et al., 1987, 2000; Peláez-Campomanes et al., 2000), así como el yacimiento de Cerro Batallones que corresponde al Vallesiense dentro ya del Mioceno Superior (Morales et al., 1992). El nuevo yacimiento de microvertebrados de Parla constituye una aportación al conocimiento de las faunas del Mioceno Medio de la "Unidad Intermedia" de Madrid con la importante nove- dad de hallarse en superposición estratigráfica con varios ricos yacimientos de flora.

Todos los taxones de micromamíferos identificados en el nuevo yacimiento son comunes en los yacimientos de la Cuenca de Madrid. Heteroxerus rubricati ha sido citado en numerosos yacimientos del Aragoniense Medio y Superior de esta cuenca (Alberdi et al., 1981; Sesé et al., 1985; Peláez-Campomanes et al., 2000). El género Armantomys, aunque muy escaso en Parla, es por lo general muy abundante en las asociaciones de micromamíferos de Madrid, a diferencia de lo que ocurre en otras cuencas como la de Calatayud durante la misma época (Sesé et al., 1985; López et al., 1987). Por último, con respecto al género Megacricetodon, tanto la especie $M$. collongensis como $M$. crusafonti están ampliamente representadas en numerosos yacimientos que corresponden respectivamente al Aragoniense Medio y Superior (Sesé et al., 1985; López et al., 1987; Peláez-Campomanes et al., 2000).

En cuanto a la adscripción taxonómica de la reducida población del género Megacricetodon de Parla, tanto por morfología como por talla puede corresponder, bien a $M$. collongensis-crusafonti o bien a M. crusafonti. Megacricetodon collongensis- crusafonti es una forma transicional entre las especies: $M$. collongensis y $M$. crusafonti, que es característica de las zonas $\mathrm{F} \mathrm{y}_{1}$, y M.crusafonti lo es de la zona $\mathrm{G}_{2}$, todas ellas del Aragoniense Superior (Daams y Freudenthal, 1988a). Desde un punto de vista biostratigráfico se puede decir por tanto que el yacimiento tiene una edad del Aragoniense Superior no final. Las zonas F, $\mathrm{G}_{1} \mathrm{y}$ $\mathrm{G}_{2}$ se corresponden grosso modo con la unidad NM 6 del Mioceno Medio (según la biozonación de Mein, 1975).

La asociación faunística de Parla es relativamente poco diversa (faltan por ejemplo los glíridos, y otros taxones de cricétidos, como el género Fahlbuschia Mein y Freudenthal, 1971, presentes sin embargo en otros yacimientos de Madrid (Sesé et al., 1985; López et al., 1987) con un taxón dominante: Megacricetodon collongensis- crusafonti, al que corresponde el $77 \%$ de los restos identificables de roedores. Estos dos hechos: la poca diversidad relativa de las faunas y la dominancia de una o muy pocas especies, ya habían sido señalados con anterioridad en las asociaciones de micromamíferos de diversos yacimientos del Aragoniense Medio y Superior del área de Madrid por Sesé et al. (1985). No es sorprendente que el taxón más abundante en Parla sea Megacricetodon collongensis- crusafonti ya que el género Megacricetodon, muy común en todas las faunas de roedores del Mioceno Medio, es especialmente abundante en general durante el Aragoniense Superior, lo que ha sido indicado en la Cuenca de Madrid y en otras cuencas por distintos autores (Sesé et al., 1985; Meulen y Daams, 1992; López et al., 2000), por lo que algunos autores como López et al. $(1987,2000)$ le atribuyen una forma de vida gregaria a semejanza de las plagas temporales que suceden en algunos roedores actuales como los microtinos. 
Desde un punto de vista paleoambiental se pueden realizar las siguientes consideraciones: Aunque según algunos autores el género Megacricetodon puede vivir en ambientes y climas variados (Weerd y Daams, 1978), otros le atribuyen un hábitat de terreno abierto, de tipo sabana o estepa cálida (López et al., 1987, 2000). El género Heteroxerus, perteneciente a la tribu Xerini, es una ardilla terrestre que vive en un hábitat abierto en un clima seco (Weerd y Daams, 1978). El glírido del género Armantomys se considera que vive también en un hábitat abierto y en un clima seco (Weerd y Daams, 1978).

La suposición de la existencia de un medio abierto y un clima relativamente árido en la Cuenca de Madrid durante el Aragoniense Superior que indican estos hallazgos ya ha sido señalada para este área por la presencia de diversas asociaciones de micromamíferos por Sesé et al. (1985) y de mamíferos en general por Alberdi et al. (1985).

\section{CONCLUSIONES}

En la sucesión estudiada de Parla se ha podido determinar una secuencia ambiental. En el tramo inferior de la sucesión se aprecia una progradación de ambientes palustres-lagunares caracterizados por praderas húmedas, bosques de ribera con Populus, Alnus, Carya y Daphnogene y zonas pantanosas de margen lacustre donde crecían Myrica, Phragmites, Esparganiáceas, Tifáceas, etc, y algas de agua dulce que va evolucionando hacia otros ambientes de abanicos aluviales con praderas abiertas de Gramíneas, matorrales, arbustos adaptados a la xericidad, Leguminosas, Anacardiáceas, etc. Estos últimos aparecen fundamentalmente en los yacimientos 1 y 2 donde los restos vegetales se encuentran casi a techo del tramo inferior en un nivel de arena fina a media, biotítica. Es en el tramo superior de la sucesión y exclusivamente en el yacimiento 6 donde aparecen los restos de Megacricetodon, Armantomys y Heteroxerus, micromamíferos fósiles propios de medios abiertos y clima seco.

Estas inferencias paleontológicas son coherentes con la presencia de dos tramos de características bastante diferenciadas. El tramo inferior está constituido fundamentalmente por lutitas laminadas con yesos intercalados que se depositarían bajo una lámina de agua de escaso espesor, que en el caso de desaparecer daría lugar a carbonatos vadosos. Este medio ha sido interpretado como un ambiente de depósito palustre-lacustre somero en un clima árido (Calvo et al., 1989). En el tramo superior, el nivel de arenas y arcillas arenosas de colores verdosos presenta intensa bioturbación vertical por raíces y ausencia de yesos intercalados en los planos de estratificación.

Se observa, una evolución ambiental en la sucesión del Aragoniense con tendencia hacia el desarrollo de áreas esteparias en el tramo superior sobre las palustres-lacustres del inferior.

\section{AGRADECIMIENTOS}

Las intervenciones arqueológicas y paleontológicas se han realizado en cumplimiento de la Ley 18/1998 de 9 de Julio de Patrimonio Histórico de la Comunidad de Madrid, estando autorizadas, gestionadas y supervisadas por la Dirección General de Patrimonio Histórico de la Consejería de Cultura y Deporte. Los autores agradecen la colaboración prestada por D. Carlos Barrio Aldea, D. Raúl Arribas Domínguez, arqueólogos y D. Emiliano Chamorro Gabaldón, Jefe de Departamento de Asistencias técnicas de CYGSA.

Por otra parte, agradecemos a D. Enrique Soto el tratamiento informático de los dibujos de los dientes, y el mapa de situación de los yacimientos, a D. Ricardo Lara por su apoyo informático y a D. Carlos Alonso (UCM-IGE) por las reproducciones fotográficas. Nuestro agradecimiento también a uno de los revisores (Nieves López-Martinez) que con sus acertados y constructivos juicios críticos ha contribuido a una mejora sustancial del trabajo y al editor porque con sus sugerencias ha mejorado la comprensión del manuscrito.

\section{BIBLIOGRAFÍA}

Alberdi, M. T., Jiménez, E., Morales, J. y Sesé, C. 1981. Moratines: Primeros Micromamíferos en el Mioceno Medio del área de Madrid. Estudios Geológicos, 37, 291-305

Alberdi, M. T., Hoyos, M., Mazo, A. V., Morales, J., Sesé, C. y Soria, D. 1985. Bioestratigrafia, paleoecología y biogeografía del Terciario de la provincia de Madrid. In: Geología y Paleontologia del Terciario Continental de la Provincia de Madrid (Coord. M.T. Alberdi). Museo de Ciencias Naturales, C.S.I.C., Madrid, 99-105.

Bernor, R., Kovar-Eder, J., Lipscomb, D., Rögl, F., Sen, S. and Tobien, H. 1988. Systematic, stratigraphic, and Paleoenvironmental contexts of first-appering Hipparion in the Vienna Basin, Austria. Journal of Vertebrate Paleontology, 8, 427-452.

Bruijn, H. de 1967. Gliridae, Sciuridae y Eomyidae (Rodentia, Mammalia) miocenos de Calatayud (provincia de Zaragoza, España) y su relación con la bioestratigrafía del área. Boletín del Instituto Geológico y Minero de Espa$\tilde{n} a$, 78, 187-373.

Calvo, J. P. 2000. Geología del Mioceno de Madrid. In: Patrimonio Paleontológico de la Comunidad de Madrid. Monografía n ${ }^{\circ}$ 6, Arqueología, Paleontología y Etnografía (coord. J. Morales). Comunidad de Madrid, Madrid, 95-101.

Calvo, J.P., Ordóñez, S., García del Cura, M.A., Hoyos, M., Alonso- Zarza, A.M., Sanz, E. y Rodríguez Aranda, J.P. 1989. Sedimentología de los complejos lacustres miocenos de la Cuenca de Madrid. Acta Geológica Hispánica, 24, 281-298.

Ceballos, L. y Ruiz de la Torre, J. 1971. Arboles y arbustos de la España penínsular. Instituto Forestal de Investigaciones y Experiencias y Escuela Técnica Superior de Ingenieros de Montes, Madrid, $512 \mathrm{pp}$.

Cuenca Bescós, G. 1988. Revisión de los Sciuridae del Ara- 
goniense y del Rambliense en la fosa de Calatayud-Montalbán. Scripta Geologica, 87, 1-116.

Daams, R. and Freudenthal, M. 1988a. Synopsis of the DutchSpanisch collaboration program int he Aragonian type area, 1975-1986. Scripta Geologica, Spec. Issue 1, 3-18.

Daams, R. and Freudenthal, M. 1988b. Cricetidae (Rodentia) from the type-Aragonian; the genus Megacricetodon. Scripta Geologica, Spec. Issue 1, 39-132.

Daams, R., Freudenthal, M. and Meulen, A. J. Van der 1988. Ecostratigraphy of Micromammal faunas from the Neogene of Spain, Scripta Geologica., Spec. Issue 1, 287-302.

Fernández López, S.R. 2000. Temas de Tafonomía. Departamento de Paleontología. Universidad Complutense de Madrid, 167 pp.

Fernández Marrón. M.T, Fonollá Ocete, J. F. y Jiménez Rodrigo, J C. 2002 Primeros datos Paleobotánicos y Palinológicos de una flora miocénica procedente de la unidad intermedia en el sector central de la cuenca de Madrid. Geogaceta, 31, 177-180.

Hably, L. and Kvacek, Z. 1998. Pliocene mesophytic forest surrounding crater lakes in estern Hungary. Review of Palaeobotany and Palynology, 101, 257-269.

Hoyos, M., Junco, F., Plaza, J.M., Ramírez, A. y Ruiz, J. 1985. El Mioceno de Madrid. In: Geología y Paleontología del Terciario Continental de la Provincia de Madrid (Coord. M.T. Alberdi). Museo de Ciencias Naturales, C.S.I.C., Madrid, 9-16.

Lomoschitz, A., Calvo, J.P. y Ordóñez, S. 1985. Sedimentología de las facies detríticas de la unidad intermedia del mioceno al sur y este de Madrid. Estudios Geológicos, 41, 343-358.

López, N., Sesé, C. y Herráez, E. 1987. Los yacimientos de Micromamíferos del área de Madrid. Boletín Geológico y Minero, 98 , 159-176.

López Martínez, N., Élez Villar, J., Hernando Hernando, M., Cavia, A. L., Mínguez Gandú, D., Polonio Martín, I., Salesa, M. J., Mazo, A. V. y Sánchez, I. M. 2000. Los vertebrados fósiles de Somosaguas (Pozuelo de Alarcón, Madrid). In: Patrimonio Paleontológico de la Comunidad de Madrid, Monografía n ${ }^{\circ}$ 6. Arqueología, Paleontología y Etnografía (Coord. J. Morales). Comunidad de Madrid, Madrid, 130-140.

Martín-Closas, C. et Picó, P. 1998. Implications de la taphonomie des plantes dans la Paléoclimatologie. Un exemple dans l'Oligocène de Sarral (Bassin de l'Ebre, Catalogne). OFP Informations, 23, 23-24.

Mein, P. 1975. Biozonation du Neogene Mediterraneen a partir des Mammiferes. IUGS, RCMNS Working group Bratislava, 78-81

Meulen, A. J van der and De Bruijn, H. 1982. The Mammals from the lower Miocene of Aliveri (Island of Evia, Greece). Part II: The Gliridae. Proceedings Koninklijke Nederlandse Akaemie van Wetenschappen, B, 5, 485-524.

Meulen, A., J. van der and Daams, R. 1992. Evolution of Early-Middle Miocene rodent faunas in relation to longterm palaeoenvironmental changes. Palaeogeography, Palaeoclimatology, Palaeoecology, 93, 227-253.

Morales, J., Capitán, J., Calvo, J. P. y Sesé, C. 1992. Nuevo yacimiento de vertebrados del Mioceno Superior al Sur de Madrid (Cerro Batallones, Torrejón de Velasco). Geogaceta, 12, 77-80.

Pais, J. 1981. Contribuiçao para o conhecimento da vegetaçao miocénica da parte ocidental da Bacia do Tejo. Tesis Doctoral. Universidade Nova de Lisboa, 328 pp. (inédito).

Peláez-Campomanes, P, Azanza, B., Calvo, J. P., Daams, R., Herráez, E., Morales, J., Nieto, M. y Soria, D. 2000. Bioestratigrafía de las faunas de mamíferos del Mioceno de Madrid: Datación de las unidades estratigráficas. In: Patrimonio Paleontológico de la Comunidad de Madrid, Monografía n ${ }^{\circ}$ 6. Arqueología, Paleontología y Etnografía (Coord. J. Morales). Comunidad de Madrid, Madrid 103-129.

Sánchez, I. , Alcalá, L., Fraile, S., Montoya, P. y Morales, J. 2000 Tafonomía. In: Patrimonio Paleontológico de la Comunidad de Madrid, Monografía n ${ }^{\circ}$ 6, Arqueología, Paleontología y Etnografía (Coord. J. Morales). Comunidad de Madrid, Madrid 140-149.

Sesé, C. 1980. Mamíferos del Mioceno Medio de Escobosa de Calatañazor (Soria). Tesis Doctoral. Universidad Complutense Madrid, 388 pp. (inédita).

Sesé, C., López, N. y Herráez, E. 1985. Micromamíferos (Insectívoros, Roedores y Lagomorfos) de la provincia de Madrid. In: Geología y Paleontología del Terciario continental de la provincia de Madrid (Coord. M.T. Alberdi). Museo de Ciencias Naturales, C.S.I.C., 29-39.

Weerd, A van der \& Daams, R. 1978. Quantitative composition of rodent faunas in the Spanish Neogene and paleoecological implications. Proceedings Koninklijke Nederlandse Akademie van Wetenschappen, B, 81, 448-473.

Manuscrito recibido: 19 de diciembre, 2002 Manuscrito aceptado: 4 de junio, 2004 